\title{
Intrinsic dipole moment on the capped carbon nanotubes
}

\author{
Minoru Otani, ${ }^{1, *}$ Susumu Okada, ${ }^{2,3}$ and Yasuharu Okamoto ${ }^{4}$ \\ ${ }^{1}$ Research Institute for Computational Sciences (RICS), National Institute of Advanced Industrial Science and Technology (AIST), \\ Umezono 1-1-1, Tsukuba 305-8568, Japan \\ ${ }^{2}$ Center for Computational Sciences and Graduate School of Pure and Applied Sciences, University of Tsukuba, \\ Tsukuba 305-8571, Japan \\ ${ }^{3}$ CREST, Japan Science and Technology Agency, 4-1-8 Honcho, Kawaguchi, Saitama 332-0012, Japan \\ ${ }^{4}$ Nano Electronics Research Laboratories, NEC Corporation, Tsukuba 305-8501, Japan
}

(Received 30 September 2009; published 28 October 2009)

\begin{abstract}
Local atomic geometry and dimensionality of the nanoscale material consisting of carbon atoms with threefold coordination are crucial for determining their electronic properties. Due to the local electronic structure difference between the pentagonal and hexagonal $\mathrm{C}$ sites, the capped carbon nanotubes are found to possess an intrinsic dipole moment of 3.5 debye around their cap region. Our first-principle total-energy calculations clearly indicate that mixed dimensionality of the capped nanotube results in the charge transfer from the nanotube to the cap region. This is a mechanism of the electron polarization of materials. Furthermore the results corroborate the importance of the interplay between the electronic and geometric structures in nanoscale materials.
\end{abstract}

DOI: 10.1103/PhysRevB.80.153413

PACS number(s): 81.05.Tp, 31.15.es, 61.46.Np, 77.84.Lf

In recent years, the low-dimensional materials consisting of carbon atoms with threefold coordination have held a premier position in nanoscale science and technology., ${ }^{1,2}$ These materials are obtained by imposing the boundary condition on graphene flakes of appropriate shapes and sizes. The condition extends the dimensionality of the carbon allotropes to one and zero dimensions. For instance, the carbon nanotube is the one-dimensional allotrope of carbon, which is described as a rolled-up graphene strip with appropriate connecting conditions between the two sides. ${ }^{3-6}$ On the other hand, fullerenes possess a hollow-cage structure with a zerodimensional network of carbon obtained by caging the appropriate number of hexagons. ${ }^{7}$ It is well-known that the difference in the boundary conditions on the nanoscale graphene sheets produces a rich variety of electronic properties. Seamless cylindrical structures result in either metallic or semiconducting properties depending on the helical arrangement of hexagonal networks on the cylinders. ${ }^{4-6}$ Furthermore, spherical harmonic electron states $\left(Y_{\mathrm{lm}}\right)$ emerge on the occupied electron states in fullerenes ${ }^{8}$ and their condensed phases exhibit super conductivity at high temperature by electron doping. ${ }^{9-12}$

The variation in the electronic structure of the carbon allotropes, depending on their dimensionality, provides a possibility for the engineering of the electronic structure of all carbon materials by mixing the allotropes with different dimensionality, size, and shape. Indeed, our previous calculation on the carbon nanopeapod, i.e., carbon nanotubes encapsulating the fullerenes, has shown that a charge transfer from metallic carbon nanotubes to the encapsulated fullerene does take place. ${ }^{13}$ As a result, the $(10,10)$ peapod is a metal with multicarriers, each of which is distributed either on the nanotube or on the chain of the fullerenes. In this case, the difference in electron negativity between the constituents results in such a charge transfer although the system is totally comprised of carbon atoms. The fullerenes, containing twelve pentagons, intrinsically possess deeper unoccupied states compared with those of the graphite and carbon nano- tubes. Thus, it is expected that the complex carbon allotropes with mixed dimensionality exhibit similar changes in their electronic properties.

The purpose of this work is to investigate the electronic structures of a carbon nanotube, which has one of its ends capped by the hemisphere of the fullerene, by studying the possibility of charge transfer between the hemisphere and the cylindrical part of the nanotube. Our first-principle totalenergy calculation has clearly shown that the capped nanotubes intrinsically possess the dipole moment around the hemisphere of about 3.5 debye. The detailed analysis of the distribution of the accumulation and the depletion of electrons indicates that the electrons are transferred from the $\mathrm{C}$ atoms belonging in the hexagons of the cylindrical part to the $\mathrm{C}$ atoms on the pentagons. Thus, the dipole moment around the hemisphere could be classified as the class of the electronic polarization that is induced by the both the dimensionality and the network topology of constituents units.

The simulation was performed using the "Simulation Tool for Atom TEchnology,"14 employing the generalized gradient approximation (GGA) with Perdew-Burke-Ernzerhof (PBE) density functional, ${ }^{15}$ Vanderbilt pseudopotential, ${ }^{16}$ and single $k$-point sampling. The valence wave functions and charge density are expanded by plane waves of up to 25 and $225 \mathrm{Ry}$, respectively. To investigate the possibility of the dipole moment on the capped nanotube, we adopted the effective screening medium (ESM) method ${ }^{17}$ in which the Poisson equation is solved by imposing the open boundary condition along the tube axis. While in the lateral direction, we adopted a supercell model in which a capped nanotube was placed with its outer wall separated from the other walls of adjacent nanotubes by $10 \AA$. Here we investigate the finite length $(10,10)$ nanotube, which has one of its ends capped by the hemisphere of $\mathrm{C}_{240}$ fullerene with $I_{\mathrm{h}}$ symmetry. On the other hand, each $\mathrm{C}$ atom at the opposite end of the nanotube is terminated by a $\mathrm{H}$ atom to mimic the infinite-length capped carbon nanotube.

Figure 1(a) shows the distribution of the accumulated and 

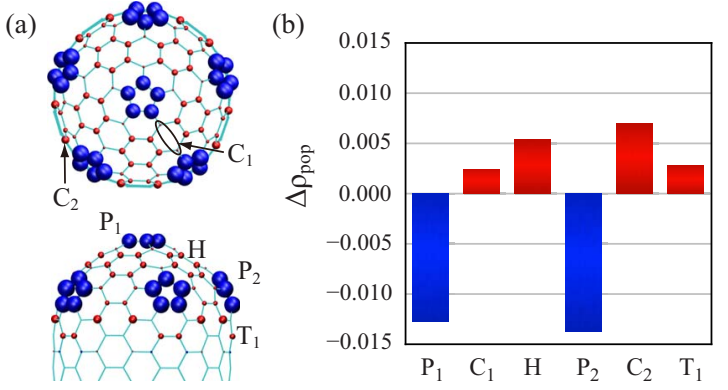

FIG. 1. (Color online) (a) Accumulated (blue/dark gray spheres) and diminished (red/gray spheres) charges of $\mathrm{C}$ atoms, (b) histogram of the charge accumulation and depletion. Radius of the spheres indicates the population of the accumulated/diminished charges. The Hirshfeld partitioning scheme (Ref. 18) is used to evaluate the population of each $\mathrm{C}$ atom.

diminished charges of the capped carbon nanotubes. Charge redistribution obviously occurs at the region of the hemisphere and the neck of the nanotube, which connects the cylinder with the hemisphere: Electrons increase at the atomic site of the pentagon, while they decrease at the $\mathrm{C}$ atoms on the hexagons in the hemisphere and the neck of the capped nanotube. This redistribution induces the dipole moment of 3.5 debye around the hemisphere of the capped carbon nanotube.

For this dipole moment, the cylindrical part of the capped nanotube connected to the hemisphere was essential. We investigated how the length of the cylindrical part of the capped nanotube affected the dipole moment. Figure 2 shows the calculated dipole moment of capped nanotubes as a function of the length of the cylindrical part. The magnitude of the moment gradually increases with increasing cylinder length. For the cylinder length of $14.7 \AA$ corresponding with the 6 units of the armchair nanotube, the moment saturated at the 3.5 debye. Thus, the results corroborate the fact that the capped nanotube intrinsically possesses a dipole moment around its cap region. The capped nanotubes may exhibit a remarkable response to the external electronic field compared with nanotubes with open ends.

It should be noted that the dipole moment already exists for the hemisphere of the $\mathrm{C}_{240}$ fullerene which has one end terminated by $\mathrm{H}$ atoms. This moment is attributed to the strong rehybridization between the $\pi$-like state and the $s$

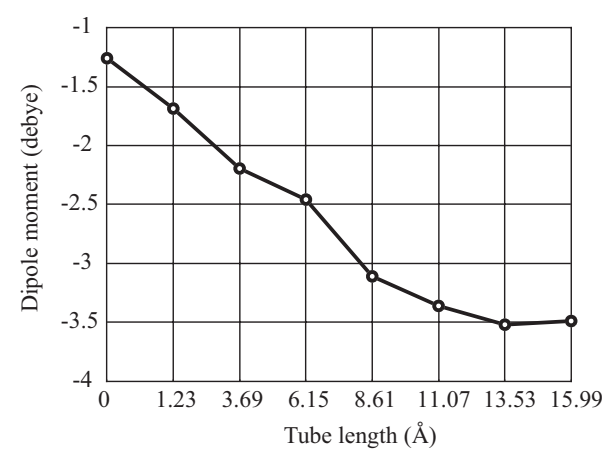

FIG. 2. Dipole moment of capped nanotubes as a function of the nanotube length. state of $\mathrm{C}$ atoms at the pentagons. The rehybridization shifts the rehybridized $\pi$-like state downward when compared with the $\pi$-like state of the $\mathrm{C}$ atoms situated in the hexagons. Thus, the charge redistribution from the hexagon to the pentagons is expected to take place in the threefold coordinate network of $\mathrm{C}$ with a hollow-cage structure. In this case, the asymmetric atomic structure of the hemisphere causes the dipole moment of 1.3 debye.

To make quantitative assessments of the charge accumulation and depletion of the capped nanotube, we analyzed the populations at each atomic site. Figure 1(b) shows the histogram of the charge population at each symmetrical individual atomic site. Accumulation mainly occurs at the $30 \mathrm{C}$ atoms belonging to the six pentagons so that the electron accumulation is considerably greater than electron depletion. The calculated amount of the accumulated charge at the $\mathrm{C}$ atoms on the pentagon is about $0.013 e$ irrespective of their mutual position in the cap. The distribution of the diminished charge exhibits significant site dependence. Around the polar region surrounding the top pentagon and the neck region connecting the hemisphere and the nanotube, the electron density significantly decreases. The number of valence electrons belonging to each $\mathrm{C}$ atom in these regions is in the range of $3.999 e$ to $3.993 e$. On the remaining $\mathrm{C}$ atoms on the hexagons, only small charge depletion occurs.

In addition to the significant charge redistribution around the cap, we find a small but sizable charge accumulation and depletion at the cylindrical region of the capped nanotube. In this region, a density oscillation occurs to screen the dipole moment induced at the cap. Thus, the electrons increase at the atomic sites adjacent to those in which electrons decrease. As a result, the electrons decrease at the adjacent site in the tubular region. The oscillation rapidly approaches zero by penetrating the cylinder region.

The dipole moment was induced by the electron redistribution in terms of the electronic structure of the constituent units of the system, i.e., the $(10,10)$ nanotube, $\mathrm{C}_{240}$ fullerene, and $\mathrm{C}_{110}$ hemisphere. Figure 3 shows the electronic structure of these constituents of the capped nanotube. The lowest unoccupied (LU) state of the hemisphere is deeper than the Fermi level of the $(10,10)$ nanotube. Thus, in the capped nanotube, we expect a charge transfer from the nanotube to the cap. The deep LU state of the $\mathrm{C}_{110}$ hemisphere is attributed to the electronic structure of $\mathrm{C}_{240}$ fullerene. As shown in Fig. 3(c), the LU state of the fullerene is close to the Fermi level of the nanotube. It was pointed out above that the LU state of fullerenes is shifted lower energy compared with the LU state of the aromatic molecules consisting solely of hexagons, owing to the existence of the pentagons which act as a topological defect in the hexagonal networks. ${ }^{19}$ Indeed, a similar charge transfer was theoretically predicted in the peapods consisting of $\mathrm{C}_{60}$ and armchair nanotubes. ${ }^{13,20}$ In that case, the electrons are transferred from nanotubes to the $t_{1 \mathrm{u}}$ state (LU state) of $\mathrm{C}_{60}$, which amounts to about 0.1 electrons per $\mathrm{C}_{60}$.

Since the capped nanotubes possess the dipole moment of about 3.5 debye around their cap, we expect that the capped nanotubes will exhibit a response to the external electronic field. Thus, it is plausible that the dipole also plays an important role in the manipulation of the nanotube. It is useful 


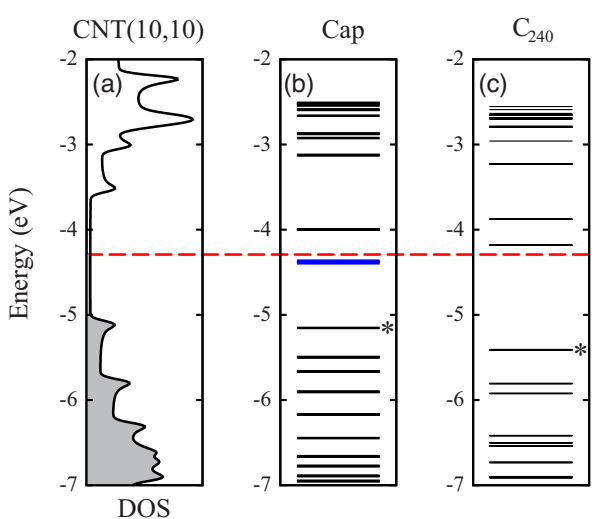

FIG. 3. (Color online) Density of state (DOS) of (a) $(10,10)$ nanotube, (b) $\mathrm{C}_{110}$ hemisphere, and (c) $\mathrm{C}_{240}$ fullerene with Ih symmetry. Energies are measured from the vacuum level defined by the energy that gives the constant value of the local potential far from the units. A horizontal dotted red line, a blue line, and the asterisk denote the Fermi level of $(10,10)$ nanotube, LU state of $\mathrm{C}_{110}$ hemisphere, and the highest occupied states of the fullerenes, respectively.

in the field of current nanotechnology. For instance, the external electronic field unravels tangled capped nanotubes. Further, the dipole-dipole interaction among the aggregates of the capped nanotube acts as a motive force for their selforganization.

In summary, we established the intrinsic dipole moment on the capped carbon nanotube based on the first-principle total-energy calculations. The calculated amount of the dipole moment is 3.5 debye concentrated at the hemisphere region of the capped nanotube. Detailed analysis of the charge density shows that the moment consists of two parts. Half of the moment originates from the charge inhomogeneity between the pentagonal and hexagonal sites in the hemisphere, which is the characteristics of the hollow-cage cluster consisting of pentagons and hexagons. The other half dipole moment is created by the charge transfer from the nanotube to the hemisphere. The mechanism of this dipole polarization is totally different from that of the usual dipole moment in molecules induced by the electronegativity difference between elements, e.g., $\mathrm{H}_{2} \mathrm{O}$ and $\mathrm{CO}$ molecules. Furthermore, the dipole moment also plays important role for nanoscale manipulations of capped nanotube.

This work was partly supported by CREST in Japan Science and Technology Agency and a grant-in-aid for scientific research from the Ministry of Education, Culture, Sports, Science and Technology of Japan. Computations were performed on NEC SX series at the University of Tsukuba, at the Yukawa Institute of Theoretical Physics, Kyoto University, at the Cybermedia Center, Osaka University, at the Information Synergy Center, Tohoku University, and at the Research Center of Computational Science, Okazaki National Institute. *minoru.otani@aist.go.jp

${ }^{1}$ M. S. Dresselhaus, G. Dresselhaus, and P. C. Eklund, Science of Fullerenes and Carbon Nanotubes (Academic Press, San Diego, 1996).

${ }^{2}$ R. Saito, G. Dresselhaus, and M. S. Dresselhaus, Physical Properties of Carbon Nanotubes (Imperial College Press, London, 1998).

${ }^{3}$ S. Iijima, Nature (London) 354, 56 (1991).

${ }^{4}$ N. Hamada, S.-I. Sawada, and A. Oshiyama, Phys. Rev. Lett. 68, 1579 (1992).

${ }^{5}$ R. Saito, M. Fujita, G. Dresselhaus, and M. S. Dresselhaus, Appl. Phys. Lett. 60, 2204 (1992).

${ }^{6}$ J. W. Mintmire, B. I. Dunlap, and C. T. White, Phys. Rev. Lett. 68, 631 (1992).

${ }^{7}$ W. Kroto, J. R. Heath, S. C. O'Brien, R. F. Curl, and R. E. Smally, Nature (London) 318, 162 (1985).

${ }^{8}$ S. Saito and A. Oshiyama, Phys. Rev. Lett. 66, 2637 (1991).

${ }^{9}$ F. Hebard, M. J. Rosseinsky, R. C. Haddon, D. W. Murphy, S. H. Glarum, T. T. M. Palstra, A. P. Ramirez, and A. R. Kortan, Nature (London) 350, 600 (1991).

${ }^{10}$ M. J. Rosseinsky, A. P. Ramirez, S. H. Glarum, D. W. Murphy,
R. C. Haddon, A. F. Hebard, T. T. M. Palstra, A. R. Kortan, S. M. Zahurak, and A. V. Makhija, Phys. Rev. Lett. 66, 2830 (1991).

${ }^{11}$ P. W. Stephens, L. Mihaly, P. L. Lee, R. L. Whetten, S. Huang, R. Kaner, F. Deiderich, and K. Holczer, Nature (London) 351, 632 (1991).

${ }^{12}$ K. Tanigaki, T. W. Ebbesen, S. Saito, J. Mizuki, J. S. Tsai, Y. Kubo, and S. Kuroshima, Nature (London) 352, 222 (1991).

${ }^{13}$ S. Okada, S. Saito, and A. Oshiyama, Phys. Rev. Lett. 86, 3835 (2001).

${ }^{14}$ Y. Morikawa, K. Iwata, and K. Terakura, Appl. Surf. Sci. 169170, 11 (2001)

${ }^{15}$ J. P. Perdew, K. Burke, and M. Ernzerhof, Phys. Rev. Lett. 77, 3865 (1996).

${ }^{16}$ D. Vanderbilt, Phys. Rev. B 41, 7892 (1990).

${ }^{17}$ M. Otani and O. Sugino, Phys. Rev. B 73, 115407 (2006).

${ }^{18}$ F. L. Hirshfeld, Theor. Chim. Acta 44, 129 (1977).

${ }^{19}$ S. Saito, S. Okada, S.-I. Sawada, and N. Hamada, Phys. Rev. Lett. 75, 685 (1995).

${ }^{20}$ M. Otani, S. Okada, and A. Oshiyama, Phys. Rev. B 68, 125424 (2003). 